\title{
Impact of final state interactions on neutrino-nucleon pion production cross sections extracted from neutrino-deuteron reaction data
}

\author{
S. X. Nakamura, ${ }^{1,2}$ H. Kamano, ${ }^{3}$ and T. Sato ${ }^{3,4}$ \\ ${ }^{1}$ University of Science and Technology of China, Hefei 230026, People's Republic of China \\ ${ }^{2}$ Laboratório de Física Teórica e Computacional (LFTC), Universidade Cruzeiro do Sul, \\ São Paulo, SP 01506-000, Brazil \\ ${ }^{3}$ Research Center for Nuclear Physics, Osaka University, Ibaraki, Osaka 567-0047, Japan \\ ${ }^{4} J-P A R C$ Branch, KEK Theory Center, IPNS, KEK, Tokai, Ibaraki 319-1106, Japan
}

(Received 4 December 2018; revised manuscript received 28 January 2019; published 20 February 2019)

\begin{abstract}
The current and near-future neutrino-oscillation experiments require significantly improved neutrinonucleus reaction models. Neutrino-nucleon pion production data play a crucial role to validate corresponding elementary amplitudes that go into such neutrino-nucleus models. Thus, the currently available data extracted from charged-current neutrino-deuteron reaction data $\left(\nu_{\mu} d \rightarrow \mu^{-} \pi N N\right)$ must be corrected for nuclear effects such as the Fermi motion and final state interactions (FSI). We study $\nu_{\mu} d \rightarrow \mu^{-} \pi N N$ with a theoretical model including the impulse mechanism supplemented by FSI from $N N$ and $\pi N$ rescatterings. An analysis of the spectator momentum distributions reveals that the FSI effects significantly reduce the spectra over the quasifree peak region and leads to a useful recipe to extract information of elementary $\nu_{\mu} N \rightarrow \mu^{-} \pi N$ processes using $\nu_{\mu} d \rightarrow \mu^{-} \pi N N$ data, with the important FSI corrections taken into account. We provide $\nu_{\mu} N \rightarrow \mu^{-} \pi N$ total cross sections by correcting the deuterium bubble chamber data for the FSI and Fermi motion. The results will bring a significant improvement on neutrino-nucleus reaction models for the near-future neutrino-oscillation experiments.
\end{abstract}

DOI: 10.1103/PhysRevD.99.031301

The current frontier of neutrino-oscillation experiments, such as the T2K [1] and the DUNE [2], is to determine the charge-parity violating phase $\left(\delta_{\mathrm{CP}}\right)$ and the neutrino mass hierarchy as the primary objective. In this era of precision neutrino experiments, we must improve the current situation that the uncertainty in our knowledge of neutrino-nucleus cross sections in the few-GeV neutrino energy region is one of the largest sources of systematic uncertainty in extracting the oscillation parameters from the data $[3,4]$.

A reliable model for the elementary neutrino-nucleon reactions is a key ingredient in neutrino-nucleus interaction generators such as NEUT [5], GENIE [6], NuWro [7], and GiBUU [8], to be used in neutrino-oscillation analyses. Many microscopic models [9] for the neutrino-nucleon single pion productions $(\nu N \rightarrow l \pi N)$ have been developed with different dynamical contents [11-13]; see Ref. [14] for an overview of these microscopic models and Ref. [15] for a detailed comparison. The common procedure in developing all the models is to adjust the dominant $\Delta(1232)$ excitation mechanism to fit the total cross section data

Published by the American Physical Society under the terms of the Creative Commons Attribution 4.0 International license. Further distribution of this work must maintain attribution to the author(s) and the published article's title, journal citation, and DOI. Funded by SCOAP.
$[16,17]$ of $\nu_{\mu} p \rightarrow \mu^{-} \pi^{+} p, \quad \nu_{\mu} n \rightarrow \mu^{-} \pi^{+} n$, and $\nu_{\mu} n \rightarrow$ $\mu^{-} \pi^{0} p$. These currently available data for $\nu_{\mu} N \rightarrow \mu^{-} \pi N$ were extracted from neutrino-deuteron reaction $\left(\nu_{\mu} d \rightarrow\right.$ $\mu^{-} \pi N N$ ) data, assuming the quasifree mechanism [Fig. 1(a)]. In order to reduce the systematic uncertainty of the neutrinooscillation parameters, an urgent task is to clarify the effects of the final state interactions (FSI), such as the nucleon and pion rescattering processes [Figs. 1(b) and 1(c)], and then correct the extracted $\nu_{\mu} N \rightarrow \mu^{-} \pi N$ cross sections.

In this paper, we analyze the spectator momentum distributions of $\nu_{\mu} d \rightarrow \mu^{-} \pi N N$, the data of which would be obtainable from a possible future neutrino-deuteron experiment [18]. Taking account of the important FSI corrections, we then find a useful recipe for extracting the information of $\nu_{\mu} N \rightarrow \mu^{-} \pi N$ processes from the deuterontarget data. We will also provide improved $\nu_{\mu} N \rightarrow \mu^{-} \pi N$ total cross sections by correcting the bubble chamber data $[19,20]$, which are free from the flux uncertainty, for the FSI and Fermi motion effects. The improved data will enable one to develop a more accurate $\nu_{\mu} N \rightarrow \mu^{-} \pi N$ model to be used in extracting the neutrino properties from the oscillation experiments of the precision era.

The first attempt towards understanding the FSI effects on $\nu_{\mu} d \rightarrow \mu^{-} \pi N N$ differential cross sections has been made recently [21], and sizable FSI effects were found in the quasifree $\Delta(1232)$-production region. However, their 


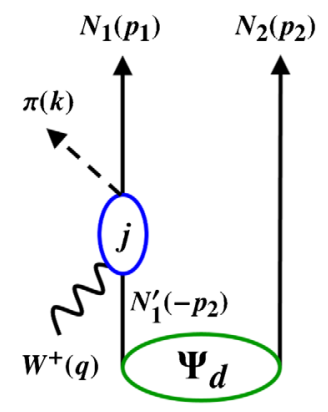

(a) Impulse

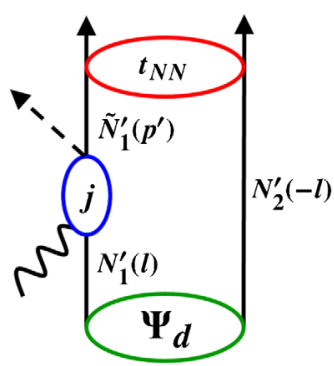

(b) NN rescattering

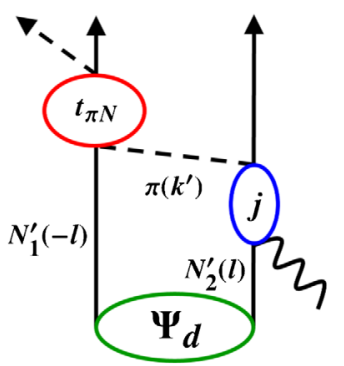

(c) $\pi \mathrm{N}$ rescattering

FIG. 1. Mechanisms in our $\nu_{\mu} d \rightarrow \mu^{-} \pi N N$ model: (a) impulse; (b) $N N$ rescattering; (c) $\pi N$ rescattering mechanisms. Particle labels and their momenta (in parentheses) are defined.

analysis focused on this particular kinematical region, and thus understanding the FSI corrections on the $\nu_{\mu} N \rightarrow \mu^{-} \pi N$ data $[16,17]$ is beyond their scope. Our calculation will cover the whole phase space of $\nu_{\mu} d \rightarrow \mu^{-} \pi N N$ with the Monte Carlo phase-space integral.

Our $\nu_{\mu} d \rightarrow \mu^{-} \pi N N$ reaction model consists of the relevant mechanisms depicted in Fig. 1: (a) impulse mechanism; (b) $N N \rightarrow N N$ FSI mechanism; (c) $\pi N \rightarrow \pi N$ FSI mechanism. We use elementary weak pion production amplitudes and $\pi N \rightarrow \pi N$ scattering amplitudes generated from a dynamical coupled-channel (DCC) model $[13,14,22,23]$. The DCC model has been developed for a unified description of the hadronic and electroweak reactions on the single nucleon in the nucleon resonance region: $\pi N, \gamma^{(*)} N \rightarrow X$ and $\nu_{l} N \rightarrow l^{-} X$ with $X=\pi N, \pi \pi N, \eta N$, $K \Lambda, K \Sigma$. The model has been extensively tested by a large amount of data ( 27000 data points) on $\pi N, \gamma N \rightarrow \pi N, \eta N$, $K \Lambda, K \Sigma[22,23]$ and also by data on electron-induced reactions [13] for $W \lesssim 2 \mathrm{GeV}$ ( $W$, invariant mass of the hadron system) and $Q^{2} \leq 3 \mathrm{GeV}^{2}$. The DCC elementary amplitudes are particularly suited for describing the neutrino-deuteron reactions that include loop diagrams of hadronic rescatterings. This is because the DCC model possesses unique features, which the other microscopic models do not, such as (i) a consistent description of the weak pion productions and $\pi N$ reactions satisfying twobody as well as three-body unitarity requirements; and (ii) off-shell amplitudes are available by construction. The latter feature is crucial for embedding the elementary amplitudes into the deuteron reaction model in a manner consistent with the multiple scattering theory. The deuteron wave function and $N N$ scattering amplitudes are generated from a realistic $N N$ potential; here we employ the CD-Bonn potential [24].

We have already studied $\gamma d \rightarrow \pi N N[25,26]$ and $\gamma d \rightarrow$ $\eta p n$ [27] using a similar DCC-based model for meson photoproductions and have shown that significant FSI effects bring model predictions into a good agreement with the data. These results validate the predictive power of our approach and allow us to estimate the FSI effects on the neutrino-induced pion productions with a good level of reliability.

The cross sections for charged-current neutrino-deuteron reactions in the laboratory frame are given as

$$
\frac{d \sigma}{d \Omega_{l^{\prime}} d E_{l^{\prime}}}=\left(\frac{G_{F} V_{u d}}{\sqrt{2}}\right)^{2} \frac{1}{4 \pi^{2}} \frac{\left|\vec{p}_{l^{\prime}}\right|}{\left|\vec{p}_{l}\right|} L^{\mu \nu} W_{\mu \nu},
$$

where $G_{F}$ and $V_{u d}$ are the Fermi coupling constant and Cabibbo-Kobayashi-Maskawa matrix element, respectively. The lepton tensor $L^{\mu \nu}$ is given with the initial neutrino $\left(p_{l}\right)$ and the final lepton $\left(p_{l^{\prime}}\right)$ momenta. The hadron tensor is defined by

$W^{\mu \nu}=\sum_{\bar{i}, f}(2 \pi)^{3} \delta^{(4)}\left(p_{i}+q-p_{f}\right)\left\langle f\left|J^{\mu}\right| i\right\rangle\left\langle f\left|J^{\nu}\right| i\right\rangle^{*}$,

where $p_{i}\left(p_{f}\right)$ is the total four-momentum of the initial (final) hadron state and $q=p_{l}-p_{l^{\prime}}$; the average (sum) over the initial (final) hadron states is denoted by $\sum_{\bar{i}}\left(\sum_{f}\right)$. The hadron current matrix element $\left\langle f\left|J^{\mu}\right| i\right\rangle$ includes the impulse, $N N$ FSI, and $\pi N$ FSI mechanisms which are given more explicitly with the particle labels and momenta defined in Fig. 1 as

$$
\begin{aligned}
\left\langle f\left|J_{\mathrm{imp}}^{\mu}\right| i\right\rangle= & \sqrt{2} \sum_{s_{1}^{\prime}, t_{1}^{\prime}}\left\langle\pi\left(\boldsymbol{k}, t_{\pi}\right) N_{1}\left(\boldsymbol{p}_{1}, s_{1}, t_{1}\right)\left|t_{\pi N, W^{+} N}\left(M_{\pi N_{1}}\right)\right| W^{+}(\boldsymbol{q}, \lambda) N_{1}^{\prime}\left(-\boldsymbol{p}_{2}, s_{1}^{\prime}, t_{1}^{\prime}\right)\right\rangle \\
& \times\left\langle N_{1}^{\prime}\left(-\boldsymbol{p}_{2}, s_{1}^{\prime}, t_{1}^{\prime}\right) N_{2}\left(\boldsymbol{p}_{2}, s_{2}, t_{2}\right) \mid \Psi_{d}\left(s_{d}\right)\right\rangle,
\end{aligned}
$$



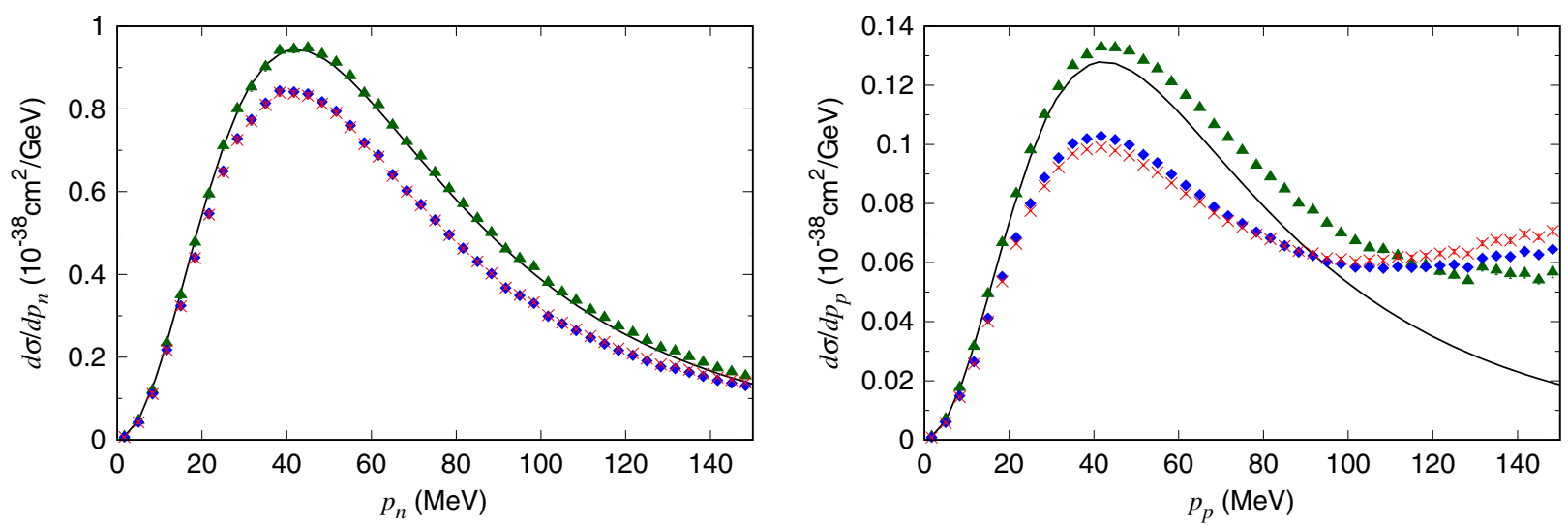

FIG. 2. Neutron (left) and proton (right) momentum distributions in $\nu_{\mu} d \rightarrow \mu^{-} \pi^{+} p n$ at $E_{\nu}=0.5 \mathrm{GeV}$. The green triangles (blue diamonds) [red crosses] are calculated with the impulse (impulse $+N N$ rescattering) [impulse $+N N+\pi N$ rescattering] mechanisms. The errors are only statistical from the Monte Carlo integral and are not shown when smaller than the point size. The black solid curve in the left [right] panel is $\nu_{\mu} p \rightarrow \mu^{-} \pi^{+} p\left[\nu_{\mu} n \rightarrow \mu^{-} \pi^{+} n\right]$ cross sections convoluted with the deuteron wave function as in Eq. (6).

$$
\begin{aligned}
\left\langle f\left|J_{N N}^{\mu}\right| i\right\rangle= & \sqrt{2} \sum_{s_{1}^{\prime}, \tilde{s}_{1}^{\prime}, s_{2}^{\prime}, t_{1}^{\prime}} \int d \boldsymbol{l}\left\langle N_{1}\left(\boldsymbol{p}_{1}, s_{1}, t_{1}\right) N_{2}\left(\boldsymbol{p}_{2}, s_{2}, t_{2}\right)\left|t_{N N, N N}\left(M_{N_{1} N_{2}}\right)\right| \tilde{N}_{1}^{\prime}\left(\boldsymbol{q}-\boldsymbol{k}+\boldsymbol{l}, \tilde{s}_{1}^{\prime}, t_{1}\right) N_{2}^{\prime}\left(-\boldsymbol{l}, s_{2}^{\prime}, t_{2}\right)\right\rangle \\
& \times \frac{\left\langle\pi\left(\boldsymbol{k}, t_{\pi}\right) \tilde{N}_{1}^{\prime}\left(\boldsymbol{q}-\boldsymbol{k}+\boldsymbol{l}, \tilde{s}_{1}^{\prime}, t_{1}\right)\left|t_{\pi N, W^{+} N}(W)\right| W^{+}(\boldsymbol{q}, \lambda) N_{1}^{\prime}\left(\boldsymbol{l}, s_{1}^{\prime}, t_{1}^{\prime}\right)\right\rangle}{E-E_{N}(\boldsymbol{q}-\boldsymbol{k}+\boldsymbol{l})-E_{N}(-\boldsymbol{l})-E_{\pi}(\boldsymbol{k})+i \epsilon}\left\langle N_{1}^{\prime}\left(\boldsymbol{l}, s_{1}^{\prime}, t_{1}^{\prime}\right) N_{2}^{\prime}\left(-\boldsymbol{l}, s_{2}^{\prime}, t_{2}\right) \mid \Psi_{d}\left(s_{d}\right)\right\rangle, \\
\left\langle f\left|J_{\pi N}^{\mu}\right| i\right\rangle= & \sqrt{2} \sum_{s_{1}^{\prime}, s_{2}^{\prime} t_{1}^{\prime}, t_{2}^{\prime}, t_{\pi}^{\prime}} \int d \boldsymbol{l}\left\langle\pi\left(\boldsymbol{k}, t_{\pi}\right) N_{1}\left(\boldsymbol{p}_{1}, s_{1}, t_{1}\right)\left|t_{\pi N, \pi N}\left(M_{\pi N_{1}}\right)\right| \pi\left(\boldsymbol{q}-\boldsymbol{p}_{2}+\boldsymbol{l}, t_{\pi}^{\prime}\right) N_{1}^{\prime}\left(-\boldsymbol{l}, s_{1}^{\prime}, t_{1}^{\prime}\right)\right\rangle \\
& \times \frac{\left\langle\pi\left(\boldsymbol{q}-\boldsymbol{p}_{2}+\boldsymbol{l}, t_{\pi}^{\prime}\right) N_{2}\left(\boldsymbol{p}_{2}, s_{2}, t_{2}\right)\left|t_{\pi N, W^{+} N}(W)\right| W^{+}(\boldsymbol{q}, \lambda) N_{2}^{\prime}\left(\boldsymbol{l}, s_{2}^{\prime}, t_{2}^{\prime}\right)\right\rangle}{E-E_{N}\left(\boldsymbol{p}_{2}\right)-E_{N}(-\boldsymbol{l})-E_{\pi}\left(\boldsymbol{q}-\boldsymbol{p}_{2}+\boldsymbol{l}\right)+i \epsilon}\left\langle N_{1}^{\prime}\left(-\boldsymbol{l}, s_{1}^{\prime}, t_{1}^{\prime}\right) N_{2}^{\prime}\left(\boldsymbol{l}, s_{2}^{\prime}, t_{2}^{\prime}\right) \mid \Psi_{d}\left(s_{d}\right)\right\rangle,
\end{aligned}
$$

and the exchange terms that can be obtained from Eqs. (3)-(5) by flipping the overall sign and interchanging all subscripts 1 and 2 for the nucleons in the intermediate and final $\pi N N$ states. Here, the deuteron state with spin projection $s_{d}$ is denoted as $\left|\Psi_{d}\left(s_{d}\right)\right\rangle ;|N(\boldsymbol{p}, s, t)\rangle$ the nucleon state with momentum $\boldsymbol{p}$ and spin and isospin projections $s$ and $t ;\left|W^{+}(\boldsymbol{q}, \lambda)\right\rangle$ the $W^{+}$boson state with momentum $\boldsymbol{q}$ and polarization $\lambda$; and $\left|\pi\left(\boldsymbol{k}, t_{\pi}\right)\right\rangle$ the pion state with momentum $\boldsymbol{k}$ and the isospin projection $t_{\pi}$. The total energy in the laboratory frame is denoted by $E$, and the energy of a particle $x$ with the mass $m_{x}$ is $E_{x}(k)=$ $\sqrt{m_{x}^{2}+k^{2}}$. The two-body invariant masses $M_{\pi N_{1}}$ and $M_{N_{1} N_{2}}$ are determined by the final states, while $W=$ $\sqrt{\left[E-E_{N}(-\boldsymbol{l})\right]^{2}-(\boldsymbol{l}+\boldsymbol{q})^{2}}$ is used for the intermediate two-body invariant mass.

The elementary amplitudes $\left\langle\pi N\left|t_{\pi N, W^{+} N}\right| W^{+} N\right\rangle$ of the weak pion production [13] and $\left\langle\pi N\left|t_{\pi N, \pi N}\right| \pi N\right\rangle$ of the pionnucleon scattering $[22,23]$ in Eqs. (3)-(5) are first generated from the DCC model in the two-body CM frame and then boosted to the laboratory frame. The same frametransformation procedure is also needed to calculate the matrix element $\left\langle N N\left|t_{N N, N N}\right| N N\right\rangle$ of $N N$ scattering [24] in
Eq. (4). The formulas for calculating these matrix elements in the laboratory frame are given in Appendix A in Ref. [25].

As in the bubble chamber experiments [16,17], we look into the spectator momentum $\left(p_{s}\right)$ distribution in $\nu_{\mu} d \rightarrow$ $\mu^{-} \pi N^{\prime} N_{s}$ ( $N_{s}$, spectator). The cross section for $\nu_{\mu} p \rightarrow$ $\mu^{-} \pi^{+} p\left(\nu_{\mu} n \rightarrow \mu^{-} \pi^{+} n\right)$ can be extracted from the $p_{n}\left(p_{p}\right)$ distribution in $\nu_{\mu} d \rightarrow \mu^{-} \pi^{+} p n$ because, in a low- $p_{n}\left(p_{p}\right)$ region, the quasifree pion production on the proton (neutron) is expected to dominate, while the neutron (proton) would hardly contribute. If this expectation is right, the spectator momentum distribution $\left(d \sigma_{\nu_{\mu} d} / d p_{s}\right)$ calculated with the impulse approximation (IA) should be approximated accurately with the $\nu_{\mu} N \rightarrow \mu^{-} \pi N^{\prime}(\equiv \alpha)$ cross section convoluted with the deuteron wave function $\left(\Psi_{d}\right)$ as

$$
\frac{d \tilde{\sigma}_{\alpha}\left(E_{\nu}\right)}{d p_{s}}=p_{s}^{2} \int d \Omega_{p_{s}} \sigma_{\alpha}\left(\tilde{E}_{\nu}\right)\left|\Psi_{d}\left(\vec{p}_{s}\right)\right|^{2}
$$

where the total cross section $\sigma_{\alpha}$ is calculated with the same $\nu_{\mu} N \rightarrow \mu^{-} \pi N^{\prime}$ amplitudes implemented in the $\nu_{\mu} d \rightarrow$ $\mu^{-} \pi N N$ model. The boosted neutrino energy $\tilde{E}_{\nu}$ is obtained 


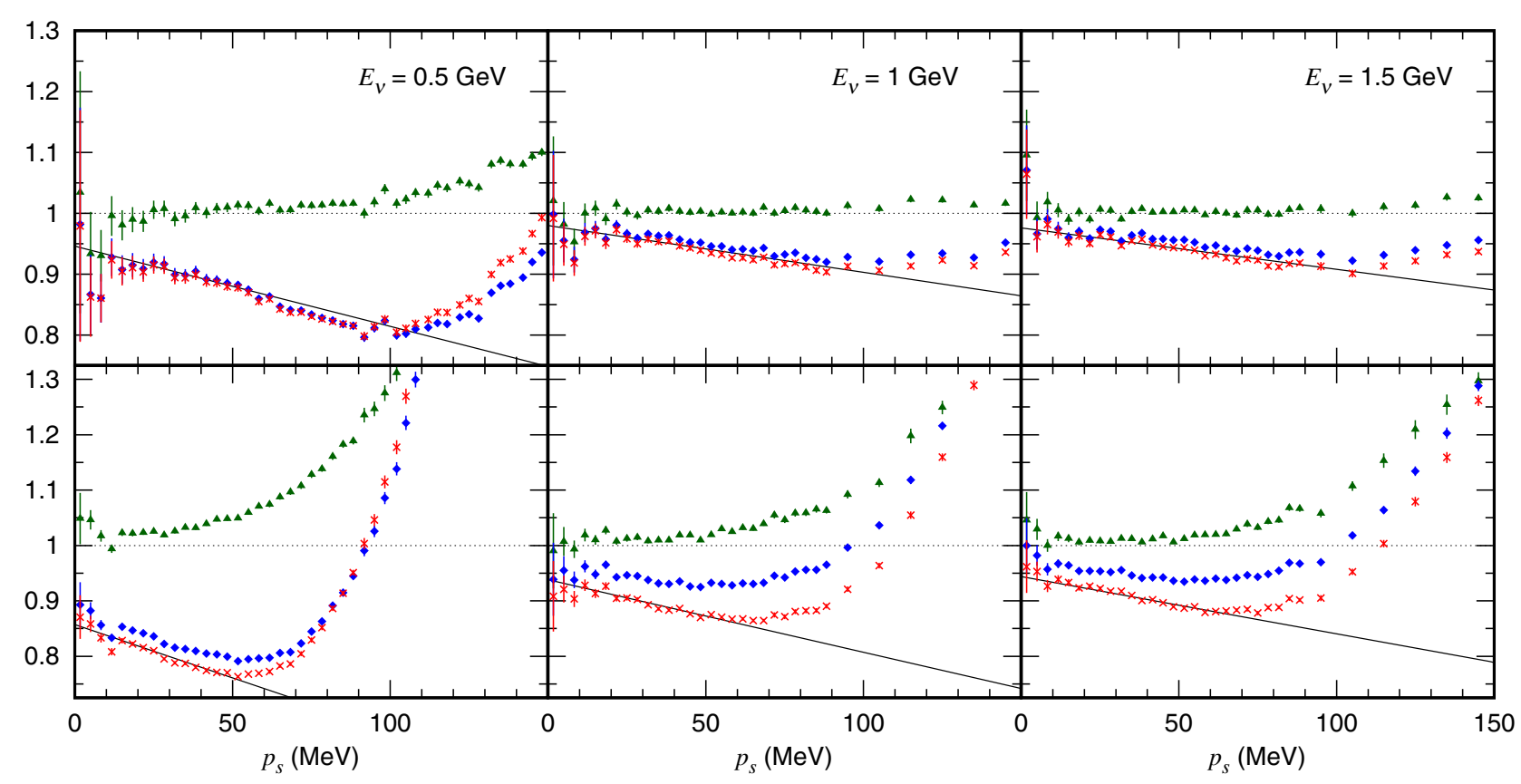

FIG. 3. Coefficients $N_{\alpha}\left(E_{\nu}, p_{s}\right)$, defined in Eq. (7), for $\nu_{\mu} d \rightarrow \mu^{-} \pi^{+} p n ; \alpha=\nu_{\mu} p \rightarrow \mu^{-} \pi^{+} p\left(\nu_{\mu} n \rightarrow \mu^{-} \pi^{+} n\right)$ and $p_{s}=p_{n}\left(p_{p}\right)$ for the upper (lower) panels. The neutrino energy is $E_{\nu}=0.5,1$, and $1.5 \mathrm{GeV}$ for the left, central, and right panels, respectively. The analytic functions $N_{\alpha}^{\text {fit }}\left(E_{\nu}, p_{s}\right)$, which are defined in Eqs. (8) and (9) and fitted to the red crosses for $p_{s} \leq 50 \mathrm{MeV}$, are also shown by the black solid lines. The other features are the same as those in Fig. 2.

from $E_{\nu}$ by the same Lorentz transformation that boosts the struck nucleon with the momentum $-\vec{p}_{s}$ to its rest.

Indeed, when the spectator in $\nu_{\mu} d \rightarrow \mu^{-} \pi^{+} p n$ is the neutron as in Fig. 2(left), where $E_{\nu}=0.5 \mathrm{GeV}$, the convoluted $\nu_{\mu} p \rightarrow \mu^{-} \pi^{+} p$ cross section (black solid curves) agrees almost perfectly with $d \sigma_{\nu_{\mu} d}^{\mathrm{IA}} / d p_{n}$ (green triangles). On the other hand, when the spectator is the proton as in Fig. 2(right), the convoluted $\nu_{\mu} n \rightarrow \mu^{-} \pi^{+} n$ cross section undershoots $d \sigma_{\nu_{\mu} d}^{\mathrm{IA}} / d p_{p}$ in the quasifree peak region. As $p_{p}$ increases, the difference between them becomes significantly larger. This difference is due to the contribution from the $\nu_{\mu} p \rightarrow \mu^{-} \pi^{+} p$ mechanism, which is responsible for $\sim 87 \%$ of the $\nu_{\mu} d \rightarrow \mu^{-} \pi^{+} p n$ total cross section from the IA calculation at this neutrino energy.

The $N N$ FSI significantly reduces $d \sigma_{\nu_{\mu} d} / d p_{s}$ for $\nu_{\mu} d \rightarrow \mu^{-} \pi^{+} p n$, especially around the quasifree peak in the low- $p_{s}$ region, as can be seen in the differences between the blue diamonds and green triangles in Fig. 2. On the other hand, the spectator momentum distribution for $\nu_{\mu} d \rightarrow \mu^{-} \pi^{0} p p$ is hardly changed by the FSI and, thus, is in good agreement with the convoluted $\nu_{\mu} n \rightarrow \mu^{-} \pi^{0} p$ cross section. The distinct differences between the $\pi^{+}$and $\pi^{0}$ productions in the FSI effects is due to the fact that the deuteron ( $p p)$ bound state can (cannot) be formed in the $\pi^{+}\left(\pi^{0}\right)$ production.

For a more quantitative study of the above observations on $d \sigma_{\nu_{\mu} d} / d p_{s}$, we define a coefficient $N_{\alpha}\left(E_{\nu}, p_{s}\right)$ by

$$
\frac{d \sigma_{\nu d}\left(E_{\nu}\right)}{d p_{s}}=N_{\alpha}\left(E_{\nu}, p_{s}\right) \frac{d \tilde{\sigma}_{\alpha}\left(E_{\nu}\right)}{d p_{s}}
$$

The predicted $N_{\alpha}\left(E_{\nu}, p_{s}\right)$ are shown in Fig. 3. A deviation from $N_{\alpha}\left(E_{\nu}, p_{s}\right) \simeq 1$ indicates the contributions from neutrino reactions on the other nucleon and/or FSI. Within the IA (green triangles), the quasifree $\nu_{\mu} p \rightarrow$ $\mu^{-} \pi^{+} p$ dominates in the low- $p_{n}$ (upper panel), while the quasifree $\nu_{\mu} n \rightarrow \mu^{-} \pi^{+} n$ dominance in the low- $p_{p}$ (lower panel) is prevented by the stronger $\nu_{\mu} p \rightarrow \mu^{-} \pi^{+} p$ channel, and thus $N_{\alpha}\left(E_{\nu}, p_{s}\right)$ quickly deviates from one.

Figure 3 also shows that the total FSI effects reduce the $p_{n}$ distribution, depending on $p_{n}$, by 10\%-20\% (5\%-10\%) for $E_{\nu}=0.5(1.5) \mathrm{GeV}$. The reduction of the $p_{p}$ distribution is even larger, and, near the quasifree peak, it is $\sim 2$ times larger than the reduction of the $p_{n}$ spectra. We also find that the FSI effect depends strongly on the neutrino energy $E_{\nu}$. The $N N$ FSI effects, which are large at small $N N$ relative energies, become smaller as $E_{\nu}$ increases. The $\pi N$ FSI effects become as large as the $N N$ FSI effect for $E_{\nu} \gtrsim 1 \mathrm{GeV}$ around the quasifree peak of the $p_{p}$ spectrum where the $\nu_{\mu} n \rightarrow \mu^{-} \pi^{+} n$ mechanism is dominant.

Once $N_{\alpha}\left(E_{\nu}, p_{s}\right)$ is provided in a simple phenomenological formula, one can easily extract $d \tilde{\sigma}_{\alpha} / d p_{s}$ of Eq. (6) from $d \sigma_{\nu d}\left(E_{\nu}\right) / d p_{s}$ data using Eq. (7) with the FSI effects taken into account. Thus, such a formula offers a useful recipe: One can determine a $\nu_{\mu} N \rightarrow \mu^{-} \pi N^{\prime}$ model by fitting the extracted $d \tilde{\sigma}_{\alpha} / d p_{s}$. Also, $N_{\alpha}\left(E_{\nu}, p_{s}\right)$ is expected to 

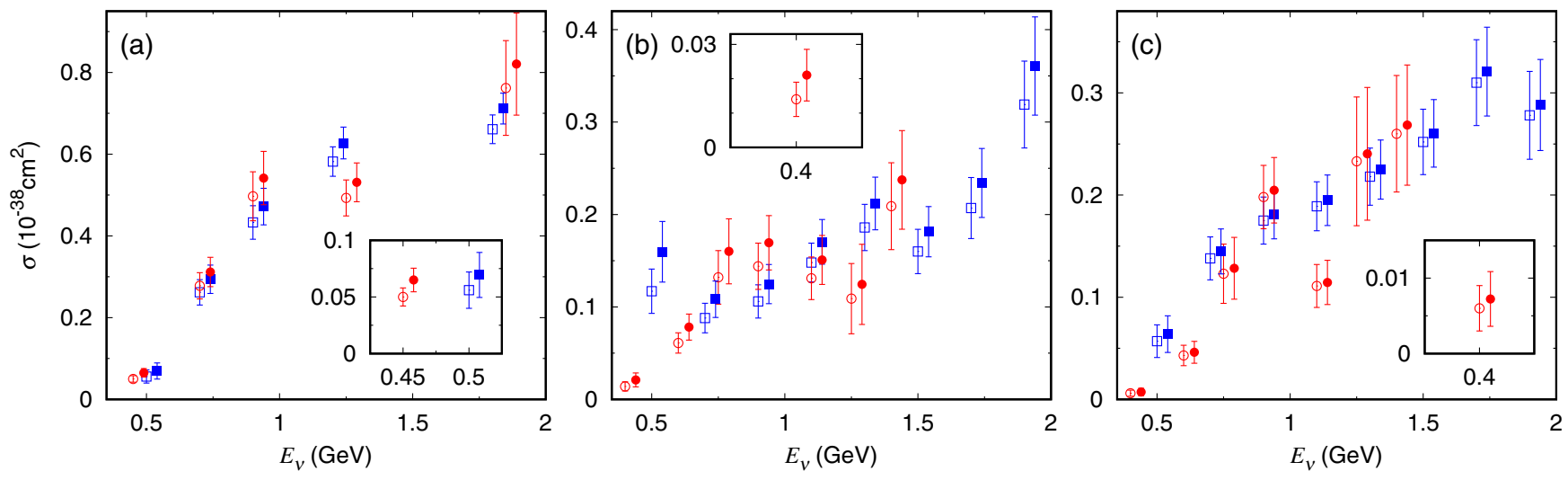

FIG. 4. Total cross sections for (a) $\nu_{\mu} p \rightarrow \mu^{-} \pi^{+} p$, (b) $\nu_{\mu} n \rightarrow \mu^{-} \pi^{+} n$, and (c) $\nu_{\mu} n \rightarrow \mu^{-} \pi^{0} p$. The open red circles (open blue squares) are the reanalyzed ANL (BNL) data [20] (no $W$ cut), which are further corrected for the Fermi motion and FSI and shown by the solid red circles (solid blue squares) slightly $[0.04 \mathrm{GeV}]$ shifted along the $E_{\nu}$ direction for better visibility. The insert shows an enlargement of the small $E_{\nu}$ region.

have a small model dependence from using our particular DCC-based model, because it is given by the ratio of $d \sigma_{\nu d} / d p_{s}$ to $d \tilde{\sigma}_{\alpha} / d p_{s}$, both of which are calculated with the same DCC elementary $\nu_{\mu} N \rightarrow \mu^{-} \pi N^{\prime}$ amplitudes. Regarding the functional form, we find it convenient to use

$$
N_{\alpha}^{\mathrm{fit}}\left(E_{\nu}, p_{s}\right)=A_{\alpha}\left(\hat{E}_{\nu}\right)+B_{\alpha}\left(\hat{E}_{\nu}\right) \hat{p}_{s},
$$

with $\hat{E}_{\nu} \equiv E_{\nu} /(1 \mathrm{GeV}), \hat{p}_{s} \equiv p_{s} /(1 \mathrm{GeV})$, and

$$
A_{\alpha}(x)=\frac{a_{\alpha} x^{2}+b_{\alpha} x+c_{\alpha}}{x^{2}+d_{\alpha} x+e_{\alpha}}, \quad B_{\alpha}(x)=\frac{f_{\alpha} x+g_{\alpha}}{x+h_{\alpha}} .
$$

We fit the parameters $\left(a_{\alpha}-h_{\alpha}\right)$ to the numerically computed $N_{\alpha}\left(E_{\nu}, p_{s}\right)$ at several neutrino energies between $E_{\nu}=0.4$ and $2 \mathrm{GeV}$ and in the $p_{s} \leq 50 \mathrm{MeV}$ region where the cross sections are dominated by the quasifree process aside from the FSI effects. The obtained parameters are presented in Table I. The fit functions are shown in Fig. 3 by the solid lines in comparison with the original numerical results.

Our finding on the FSI effects requires a modification of the flux-corrected bubble chamber data $[19,20]$. The data for $d \sigma_{\nu d} / d p_{s}$ from the $\nu d$ experiments $[16,17]$ are unavailable by now. Therefore, we use the $\nu d$ cross section

TABLE I. Parameters for $N_{\alpha}^{\mathrm{fit}}\left(E_{\nu}, p_{s}\right)$ defined in Eqs. (8) and (9).

\begin{tabular}{lccc}
\hline \hline$\alpha$ & $\nu_{\mu} p \rightarrow \mu^{-} \pi^{+} p$ & $\nu_{\mu} n \rightarrow \mu^{-} \pi^{+} n$ & $\nu_{\mu} n \rightarrow \mu^{-} \pi^{0} p$ \\
\hline$a_{\alpha}$ & 0.9498 & 0.9348 & 0.9930 \\
$b_{\alpha}$ & -0.5632 & -0.7111 & -1.035 \\
$c_{\alpha}$ & 0.2788 & 0.2211 & 0.3142 \\
$d_{\alpha}$ & -0.6381 & -0.7832 & -1.047 \\
$e_{\alpha}$ & 0.3171 & 0.2574 & 0.3188 \\
$f_{\alpha}$ & -0.5496 & -0.3170 & -25.18 \\
$g_{\alpha}$ & 0.0087 & -1.387 & -67.88 \\
$h_{\alpha}$ & -0.2983 & 0.3030 & 378.5 \\
\hline \hline
\end{tabular}

in the quasifree peak region, which includes a large portion of the total events, to estimate the corrections due to the FSI effects. We integrate Eq. (7) with respect to $p_{s}$ up to $p_{s}^{\max }=90 \mathrm{MeV}$ and then introduce an effective coefficient $\bar{N}_{\alpha}\left(E_{\nu}\right)$ to satisfy the following equation:

$$
\bar{N}_{\alpha}\left(E_{\nu}\right) \sigma_{\alpha}\left(E_{\nu}\right)=\frac{1}{A} \int_{0}^{p_{s}^{\max }} d p_{s} \frac{d \sigma_{\nu d}\left(E_{\nu}\right)}{d p_{s}}
$$

with $A \equiv \int_{0}^{p_{s}^{\max }} d^{3} p_{s}\left|\Psi_{d}\left(\vec{p}_{s}\right)\right|^{2}$. The effective coefficient $\bar{N}_{\alpha}\left(E_{\nu}\right)$ can be interpreted as an average of $N_{\alpha}\left(E_{\nu}, p_{s}\right)$, weighted with the nucleon momentum distribution in the deuteron, over $p_{s}$. We may identify $\bar{N}_{\alpha}\left(E_{\nu}\right) \sigma_{\alpha}\left(E_{\nu}\right)$ as the reanalyzed bubble chamber data $\left[\sigma_{\alpha}^{\text {data }}\left(E_{\nu}\right)\right][19,20]$, because both of them can be regarded as the effective $\nu N$ cross sections extracted from $\sigma_{\nu d}\left(E_{\nu}\right)$ without correcting for the FSI and the Fermi motion. Because the factor $\bar{N}_{\alpha}\left(E_{\nu}\right)$ distorts the true $\nu N$ cross sections $\left[\sigma_{\alpha}\left(E_{\nu}\right)\right]$ by including the FSI and Fermi motion effects, the corrected data are given by $\left[\bar{N}_{\alpha}\left(E_{\nu}\right)\right]^{-1} \sigma_{\alpha}^{\text {data }}\left(E_{\nu}\right)$. The corrected data are shown in Fig. 4 [28] in comparison with the original ones [20] to which no $W$ cut has been applied. The correction is larger for smaller $E_{\nu}$ and enhances the cross sections by scaling factors of 1.05-1.12, 1.10-1.27, and 1.01-1.02 for $\nu_{\mu} p \rightarrow \mu^{-} \pi^{+} p$, $\nu_{\mu} n \rightarrow \mu^{-} \pi^{+} n$, and $\nu_{\mu} n \rightarrow \mu^{-} \pi^{0} p$, respectively.

In summary, we have studied for the first time $\nu_{\mu} d \rightarrow$ $\mu^{-} \pi N N$ over the whole phase space with a reaction model including the FSI mechanisms. The FSI is found to significantly reduce the spectator momentum distributions, depending on the proton or neutron momentum, $\pi^{+} / \pi^{0}$ production, and neutrino energy. We have proposed a recipe to determine an elementary $\nu_{\mu} N \rightarrow \mu^{-} \pi N$ model by using data for $d \sigma_{\nu d}\left(E_{\nu}\right) / d p_{s}$ which could be obtained from possible future deuteron-target experiments. We also presented the $\nu_{\mu} N \rightarrow \mu^{-} \pi N$ total cross sections by 
correcting the flux-corrected bubble chamber data [20] for the FSI and Fermi motion. Because the bubble chamber data are currently the most important information for studying the elementary neutrino-induced pion production mechanisms, the corrected data pave the way to implementing a significantly improved pion production mechanism into a neutrino-nucleus reaction model for the nearfuture neutrino-oscillation experiments. An extension of the present analysis to differential cross sections such as $W$ and $Q^{2}$ dependences will be presented elsewhere.

The authors thank T.-S. H. Lee for carefully reading the manuscript and giving useful comments. They also thank C. Wilkinson for providing numerical values of the reanalyzed ANL and BNL data. This work is in part supported by National Natural Science Foundation of China (NSFC) under Contract No. 11625523, by Fundação de Amparo à Pesquisa do Estado de São Paulo (FAPESP), Process No. 2016/15618-8, and by JSPS KAKENHI Grants No. 25105010, No. 16K05354, and No. 18K03632. Numerical computations in this work were carried out with SR16000 at YITP in Kyoto University, the High Performance Computing system at RCNP in Osaka University, the National Energy Research Scientific Computing Center, which is supported by the Office of Science of the U.S. Department of Energy under Contract No. DE-AC02-05CH11231, and the use of the Bebop and Blues clusters in the Laboratory Computing Resource Center at Argonne National Laboratory.
[1] http://t2k-experiment.org.

[2] http://www.dunescience.org.

[3] L. Alvarez-Ruso et al., Prog. Part. Nucl. Phys. 100, 1 (2018).

[4] K. Mahn, C. Marshall, and C. Wilkinson, Annu. Rev. Nucl. Part. Sci. 68, 105 (2018).

[5] Y. Hayato, Acta Phys. Pol. B 40, 2477 (2009).

[6] C. Andreopoulos et al., Nucl. Instrum. Methods Phys. Res., Sect. A 614, 87 (2010).

[7] T. Golan, J. T. Sobczyk, and J. Zmuda, Nucl. Phys. B, Proc. Suppl. 229-232, 499 (2012).

[8] O. Buss, T. Gaitanos, K. Gallmeister, H. van Hees, M. Kaskulov, O. Lalakulich, A. B. Larionov, T. Leitner, J. Weil, and U. Mosel, Phys. Rep. 512, 1 (2012).

[9] An alternative approach, applicable only at $Q^{2} \sim 0$, is to use the PCAC relation [10].

[10] H. Kamano, S. X. Nakamura, T.-S. H. Lee, and T. Sato, Phys. Rev. D 86, 097503 (2012).

[11] D. Rein and L. M. Sehgal, Ann. Phys. (N.Y.) 133, 79 (1981); D. Rein, Z. Phys. C 35, 43 (1987); O. Lalakulich, E. A. Paschos, and G. Piranishvili, Phys. Rev. D 74, 014009 (2006); E. Hernández, J. Nieves, and M. Valverde, Phys. Rev. D 76, 033005 (2007); T. Leitner, O. Buss, L. AlvarezRuso, and U. Mosel, Phys. Rev. C 79, 034601 (2009); B. D. Serot and X. Zhang, Phys. Rev. C 86, 015501 (2012); L. Alvarez-Ruso, S. K. Singh, and M. J. Vicente Vacas, Phys. Rev. C 59, 3386 (1999); E. Hernández, J. Nieves, M. Valverde, and M. J. Vicente Vacas, Phys. Rev. D 81, 085046 (2010); M. Rafi Alam, M. Sajjad Athar, S. Chauhan, and S. K. Singh, Int. J. Mod. Phys. E 25, 1650010 (2016).

[12] T. Sato, D. Uno, and T.-S. H. Lee, Phys. Rev. C 67, 065201 (2003).
[13] S. X. Nakamura, H. Kamano, and T. Sato, Phys. Rev. D 92 , 074024 (2015).

[14] S. X. Nakamura, H. Kamano, Y. Hayato, M. Hirai, W. Horiuchi, S. Kumano, T. Murata, K. Saito, M. Sakuda, T. Sato, and Y. Suzuki, Rep. Prog. Phys. 80, 056301 (2017).

[15] J. E. Sobczyk, E. Hernández, S. X. Nakamura, J. Nieves, and T. Sato, Phys. Rev. D 98, 073001 (2018).

[16] S. J. Barish et al., Phys. Rev. D 19, 2521 (1979); G. M. Radecky et al., Phys. Rev. D 25, 1161 (1982).

[17] T. Kitagaki et al., Phys. Rev. D 34, 2554 (1986).

[18] http://www.int.washington.edu/PROGRAMS/18-2a/18-2a_ workshop.html.

[19] C. Wilkinson, P. Rodrigues, S. Cartwright, L. Thompson, and K. McFarland, Phys. Rev. D 90, 112017 (2014).

[20] P. Rodrigues, C. Wilkinson, and K. McFarland, Eur. Phys. J. C 76, 474 (2016).

[21] J.-J. Wu, T. Sato, and T.-S. H. Lee, Phys. Rev. C 91, 035203 (2015).

[22] H. Kamano, S. X. Nakamura, T.-S. H. Lee, and T. Sato, Phys. Rev. C 88, 035209 (2013).

[23] H. Kamano, S. X. Nakamura, T.-S. H. Lee, and T. Sato, Phys. Rev. C 94, 015201 (2016).

[24] R. Machleidt, Phys. Rev. C 63, 024001 (2001).

[25] S. X. Nakamura, H. Kamano, T.-S. H. Lee, and T. Sato, arXiv:1804.04757v2.

[26] S. X. Nakamura, Phys. Rev. C 98, 042201(R) (2018).

[27] S. X. Nakamura, H. Kamano, and T. Ishikawa, Phys. Rev. C 96, 042201(R) (2017).

[28] See Supplemental Material at http://link.aps.org/ supplemental/10.1103/PhysRevD.99.031301 for the numerical values of the corrected cross sections. 